\title{
Design of high efficiency achromatic metalens with large operation bandwidth using bilayer architecture
}

\author{
Yilin Wang ${ }^{1}$, Qingbin Fan ${ }^{1}$ and Ting $\mathrm{Xu}^{1,2 *}$
}

\begin{abstract}
Achromatic metalens composed of arrays of subwavelength nanostructures with spatially varying geometries is attractive for a number of optical applications. However, the limited degree of freedom in the single layer achromatic metasurface design makes it difficult to simultaneously guarantee the sufficient phase dispersion and high diffraction efficiency, which restricts the achromatic bandwidth and efficiency of metalens. Here we propose and demonstrate a high efficiency achromatic metalens with diffraction-limited focusing capability at the wavelength ranging from $1000 \mathrm{~nm}$ to $1700 \mathrm{~nm}$. The metalens comprises two stacked nanopillar metasurfaces, by which the required focusing phase and dispersion compensation can be controlled independently. As a result, in addition to the large achromatic bandwidth, the averaged focusing efficiency of the bilayer metalens is higher than $64 \%$ at the near-infrared region. Our design opens up the possibility to obtain the required phase dispersion and efficiency simultaneously, which is of great significance to design broadband metasurface-based optical devices.
\end{abstract}

Keywords: metalens; metasurface; nanostructure; waveguide

Wang YL, Fan QB, Xu T. Design of high efficiency achromatic metalens with large operation bandwidth using bilayer architecture. Opto-Electron Adv 4, 200008 (2021).

\section{Introduction}

Dispersion, as one of the most fundamental properties of optical materials, leads to a spatial separation of different wavelengths. As a result, conventional refractive optical components, such as glass lens, always have chromatic aberration. Although such chromatic aberration can be used for spectrometry, it will significantly degrade the image quality in many imaging-related applications. For the correction of chromatic aberration in multi-wavelengths applications, traditional strategy is to integrate different dispersive materials to form an apochromatic or super-achromatic lens system ${ }^{1}$. Nevertheless, this approach inevitably adds weight, complexity and cost to optical devices, which further restricts their applications in some ultracompact systems.
In recent years, metasurface, which is comprised of arrays of subwavelength nanoscatterers with spatially varying geometries, has shown excellent ability to shape the electromagnetic field at will by manipulating its amplitude, phase, and polarization ${ }^{2-12}$. Ultra-compact planar architecture, ease-of-fabrication and high diffraction efficiency make metasurface the excellent candidate for various applications such as holograms ${ }^{13-19}$, metalenses ${ }^{20-22}$, and polarimeters ${ }^{23,24}$. However, metasurface-based functional elements are classified as diffractive devices, which possess severe chromatic aberrations and thus limit their broadband optical operations. In this context, several pioneering researches have been proposed in terms of eliminating the chromatic aberrations of metalens ${ }^{25-39}$. For example, metasurface unit-cells designed for several

${ }^{1}$ National Laboratory of Solid-State Microstructures, Key Laboratory of Intelligent Optical Sensing and Integration and College of Engineering and Applied Sciences, Nanjing University, Nanjing 210093, China; ${ }^{2}$ Collaborative Innovation Center of Advanced Microstructures, Nanjing University, Nanjing 210093, China.

*Correspondence: T Xu, E-mail: xuting@nju.edu.cn

Received: 15 March 2020; Accepted: 27 April 2020; Published: 27 January 2021 
discrete operation wavelengths can be multiplexed or stacked to achieve multi-wavelengths, narrow-band achromatic metalenses ${ }^{25-31}$. In addition, based on the integrated-resonant unit elements to compensate the phase dispersion, broadband achromatic metalenses have also been demonstrated ${ }^{32-40}$. Nevertheless, the limited degree of freedom in the single layer achromatic metasurface design makes it difficult to simultaneously guarantee the sufficient phase dispersion and diffraction efficiency. As a result, the operation bandwidth and averaged efficiency of the single layer achromatic metalens are typically smaller than $40 \%$ relative to central wavelength and lower than $50 \%$, respectively.

In this work, we propose a new approach to design high efficiency achromatic metalens with large operation bandwidth based on a stacked bilayer architecture. In contrast to the conventional single layer metasurface, two layers of nanostructures in the bilayer configuration are designed to manipulate phase profile and phase dispersion respectively, which would significantly improve the operation bandwidth and efficiency while giving more options for the design. As a proof-of-concept demonstration, we design a Si bilayer metalens working at the near-infrared region, achieving a large continuous achromatic wavelength from $1000 \mathrm{~nm}$ to $1700 \mathrm{~nm}$, about $52 \%$ operation bandwidth relative to the central wavelength. In addition, the bilayer Si metalens has an averaged diffraction efficiency about $80 \%$ at the near-infrared region, and the focusing efficiency at the central wavelength of $1350 \mathrm{~nm}$ reaches up to $75 \%$. Our design opens up the possibility to overcome the challenge of improving phase dispersion and efficiency at the same time, which is of great significance to the broadband applications of meta-devices.

\section{Design of the bilayer achromatic metalens}

To realize a high-efficiency achromatic metalens, we use a tightly spaced bilayer metasurfaces architecture, as shown in Fig. 1. For the design of the top layer, the geometric phase is employed to impose a phase profile $\varphi_{\text {top }}\left(\lambda_{\max }, x, y\right)$ on transmitted waves. The generated phase profile only depends on the orientation of a waveplate-like birefringent rectangular nanopillar, which is insensitive to the wavelength. The bottom layer of the device, composed of cylindrical nanopillars with different diameters, imposes the propagation phase on incident light. The propagation phase modulation is a function of operating wavelength and can provide the appro- priate phase dispersion $\Delta \varphi(\lambda, x, y)$ to compensate phase difference between various working wavelengths. It should note that the propagation phase modulation also introduces an additional phase profile $\varphi_{\text {add }}(\lambda, x, y)$ that would affect the convergence of the incident light. Therefore, the required focusing phase profile $\varphi_{f}(\lambda, x, y)$ consists of the geometric phase modulation of the top layer and the propagation phase modulation of the bottom layer. For the case of operating wavelength $\left\{\lambda_{\min }, \lambda_{\max }\right\}$, the phase profile $\varphi_{\mathrm{am}}(\lambda, x, y)$ of the whole achromatic metalens can be expressed as:

$$
\varphi_{\mathrm{am}}(\lambda, x, y)=\varphi_{f}(\lambda, x, y)+\Delta \varphi(\lambda, x, y),
$$

with

$$
\varphi_{f}(\lambda, x, y)=\varphi_{\text {top }}\left(\lambda_{\max }, x, y\right)+\varphi_{\text {add }}(\lambda, x, y),
$$

and

$$
\Delta \varphi(\lambda, x, y)=-2 \pi\left(\sqrt{x^{2}+y^{2}+f^{2}}-f\right)\left(\frac{1}{\lambda}-\frac{1}{\lambda_{\max }}\right) .
$$

The phase dispersion is only determined by the propagation phase modulation of the bottom layer. The phase profile $\varphi_{\text {add }}(\lambda, x, y)$ should keep at a relatively low value by optimizing the structure parameters so that the phase profile $\varphi_{f}(\lambda, x, y)$ is mainly determined by the geometry phase modulation of the top layer. As a result, in contrast to the single layer achromatic metalens, the proposed bilayer metasurface architecture allows simultaneous realization of broadband response with a large bandwidth and an improved efficiency due to its capability of providing the phase profile and phase dispersion independently.

As a proof-of-concept demonstration, here we use $\mathrm{Si}$ nanopillar arrays to design an achromatic bilayer metalens working at near-infrared region. As shown in Fig. 1, each unit cell of the bilayer metalens contains two Si nanopillars. As mentioned before, the top rectangular nanopillar is design to provide basic phase by tailoring the orientations of structure and the bottom cylindrical nanopillar is design to provide phase dispersion by tailoring the diameters of structure, which can ensure large phase dispersion compensation and high efficiency. Considering the feasibility of nanofabrication, the bottom nanopillar is immersed in a polymer photoresist SU8 while the top one is exposed to the air with the gap between them $g=400 \mathrm{~nm}$. The heights for top and bottom nanopillars are $h_{1}=850 \mathrm{~nm}$ and $h_{2}=1500 \mathrm{~nm}$, respectively, with a same square lattice constant $P=500$ $\mathrm{nm}$. For incident circularly polarized light, as high 

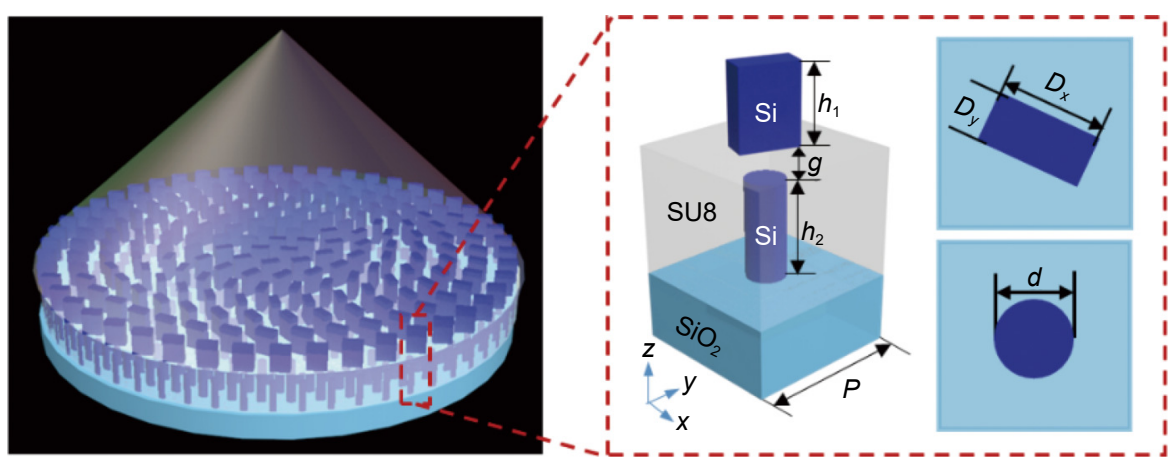

Fig. 1 | Schematic for bilayer broadband achromatic metalens. It has the same focal plane over a large continuous wavelength region. Inset: Oblique view of a unit cell of a bilayer Si nanopillars with different heights $h_{1}=850 \mathrm{~nm}, h_{2}=1500 \mathrm{~nm}$, in-plane cross-sectional dimensions $D_{\mathrm{x}}=420 \mathrm{~nm}$, $D_{\mathrm{y}}=190 \mathrm{~nm}$ and lattice constant $P=500 \mathrm{~nm}$, on a $\mathrm{SiO}_{2}$ substrate. Right: The top view of each layer.
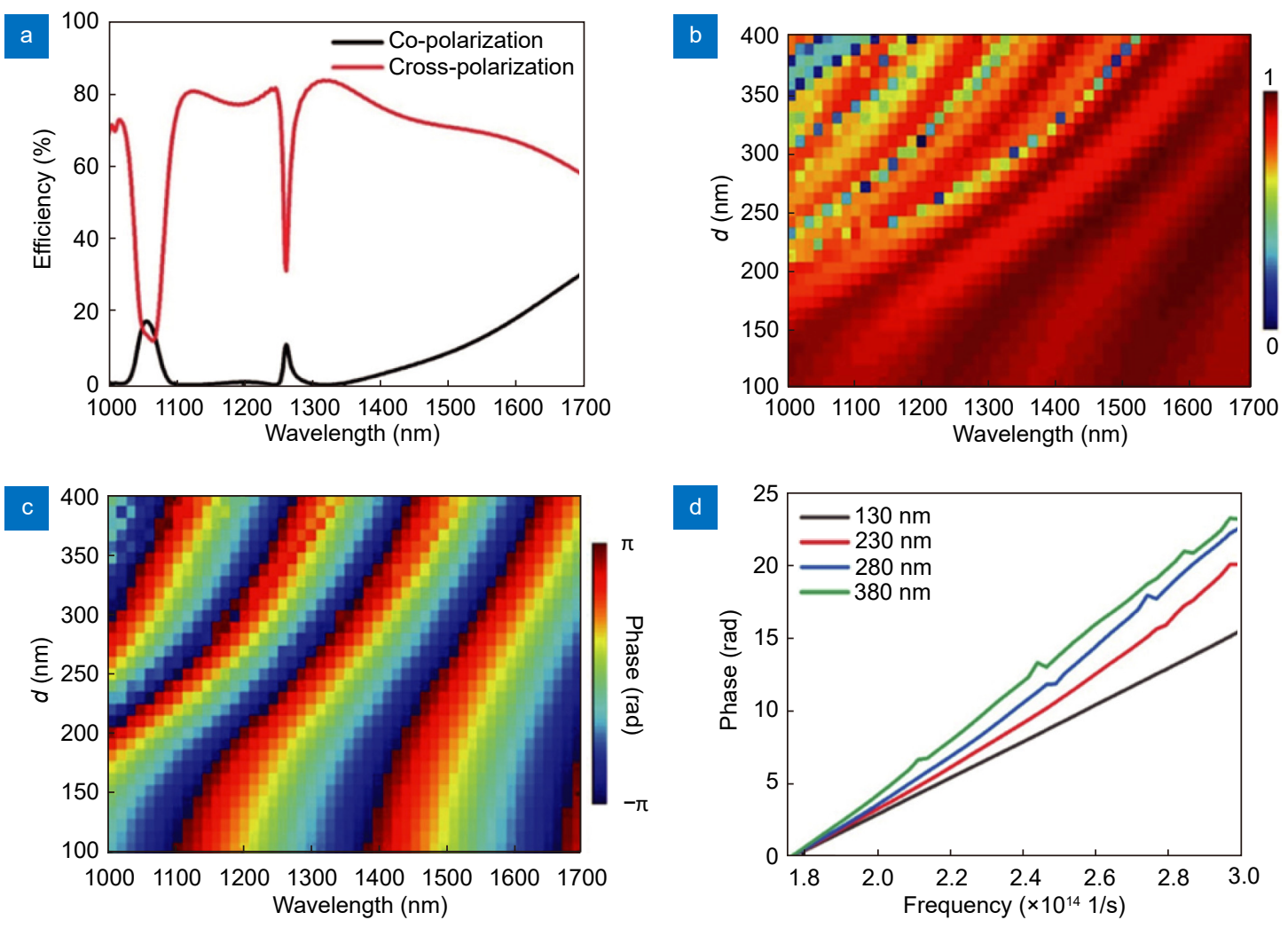

Fig. 2 | (a) Simulated polarization conversion efficiency of the top rectangular nanopillar as a function of wavelengths from $1000 \mathrm{~nm}$ to $1700 \mathrm{~nm}$. Each nanopillar with $500 \mathrm{~nm}$ periods, in-plane cross-sectional dimensions $D_{\mathrm{x}}=420 \mathrm{~nm}, D_{\mathrm{y}}=190 \mathrm{~nm}$. (b, c) Transmission coefficient and phase map of the bottom cylindrical nanopillar with different diameters $d$ as a function of wavelength from $1000 \mathrm{~nm}$ to $1700 \mathrm{~nm}$. (d) Phase spectra for cylindrical nanopillar with four different diameters as a function of frequency.

cross-polarization conversion efficiency is essential for the realization of efficient geometry phase modulation, here we pick up the optimized structural parameters for long and short axis lengths $D_{x}=420 \mathrm{~nm}$ and $D_{y}=190$ $\mathrm{nm}$ for the top rectangular nanopillar. As shown in Fig. 2(a), the calculated cross-polarization conversion efficiency of the rectangular nanopillar is relatively high across the near-infrared wavelength range from $1000 \mathrm{~nm}$ to $1700 \mathrm{~nm}$ expect for two resonant positions. As a res- ult, by changing the orientation angle of rectangular nanopillar, it can readily provide $0-2 \pi$ geometry phase modulation.

On the other side, for the bottom cylindrical nanopillar, Figs. 2(b) and 2(c) respectively illustrate the calculated transmission coefficient and propagation phase as a function of its diameter $d$ at near-infrared wavelength region. From Fig. 2(b), it can be clearly seen that the cylindrical nanopillars with different diameters always keep 
a high transmission coefficient in the broad spectral range, which is a prerequisite for achieving high efficiency lens. Besides the high transmission coefficient, the Si cylindrical nanopillar with different diameters also exhibits smooth and large phase modulation coverage at the wavelength ranging from $1000 \mathrm{~nm}$ to $1700 \mathrm{~nm}$ (Fig. 2(c)). From these results, eleven Si cylindrical nanopillars with different diameters are selected to constitute the metasurface. The phase spectra of the four structures are shown in Fig. 2(d). We need to choose smooth and linear structure of the phase spectra to achieve the function of achromatic aberration. The slope in phase spectra also can intuitively reflect the magnitude of phase dispersion. The above method is able to provide sufficient phase dispersion compensation for the design of broadband achromatic metalens. In addition, due to the employment of Si with high refractive index, the coupling effect between neighboring nanopillars is very weak, and thus each nanopillar can be regarded as an isolated waveguide. This makes the phase design for each nanopillar element stay accurate even when they are arranged in a square lattice to form the metalens.

Based on the above approach, we design and demonstrate a bilayer broadband achromatic metalens with $D=$ $100 \mu \mathrm{m}$ and $f=340 \mu \mathrm{m}$ working at near-infrared wavelength region. The top $\mathrm{Si}$ rectangular nanopillars have the same lengths of long and short sides of $D_{x}=$ $420 \mathrm{~nm}$ and $D_{y}=190 \mathrm{~nm}$ but with different orientation angle $\theta$. The bottom Si cylindrical nanopillars are designed with the diameter ranging from $130 \mathrm{~nm}$ to $380 \mathrm{~nm}$. The ideal phase profile is given in Fig. 3(a), which can theoretically achieve a perfect focusing and is mainly provided by the top layer. As expected, different phase dispersion compensation can be obtained at the different wavelengths. Fig. 3(b) reveals the bottom layer normalized phase compensation profile at the different wavelengths while it introduces an additional focusing phase. To mitigate the effect of propagation phase on convergence, we expect the $\varphi_{\text {add }}(\lambda, x, y)$ to be as small as possible under the condition of satisfying the phase dispersion, and thus the optimized phase profile of top layer becomes as shown in Fig. 3(c).

\section{Characterization of bilayer achromatic metalens}

For intuitively exhibiting the achromatic characteristics, bilayer achromatic metalens and single layer chromatic metalens with same diameter and focal length are simultaneously numerically investigated with a right-handed circularly polarized light. Figs. 4(a) and 4(b) denote their intensity profiles simulated along $x-z$ plane over a wavelength region from $1000 \mathrm{~nm}$ to $1700 \mathrm{~nm}$, at a step of $100 \mathrm{~nm}$. In contrast to the chromatic metalens exhibiting a large focal length shift similar to a Fresnel lens with increased incident wavelength (Fig. 4(a)), the bilayer chromatic metalens can converge the incident light with similar focal length at these wavelengths (Fig. 4(b)). Figs. 4(c) and 4(d) shows the simulated focal spot and corresponding cross-section of the intensity profiles at the focal plane, respectively. It can be clearly seen that the focal spot has a circularly symmetric shape and the crosssection exhibits an Airy disk distribution with low side lobes, which demonstrate the good lensing quality of the designed bilayer achromatic metalens.

In order to quantitatively reveal the performance of
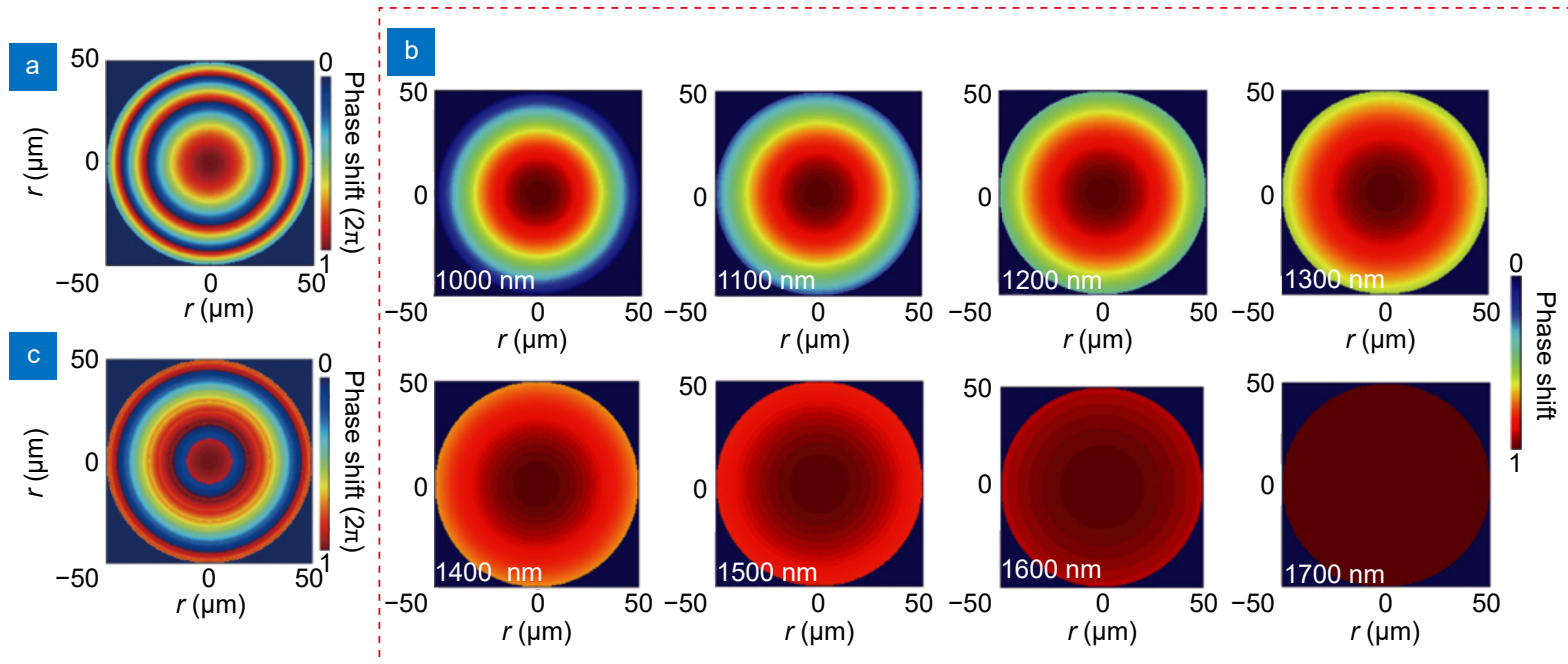

Fig. 3 | The phase profile of bilayer achromatic metalens. (a) The ideal phase profile at the $\lambda_{\max }$ (b) The bottom layer normalized phase compensation profile over a wavelength region from $1000 \mathrm{~nm}$ to $1700 \mathrm{~nm}$, at a step of $100 \mathrm{~nm}$. (c) The required phase profile of top layer. 


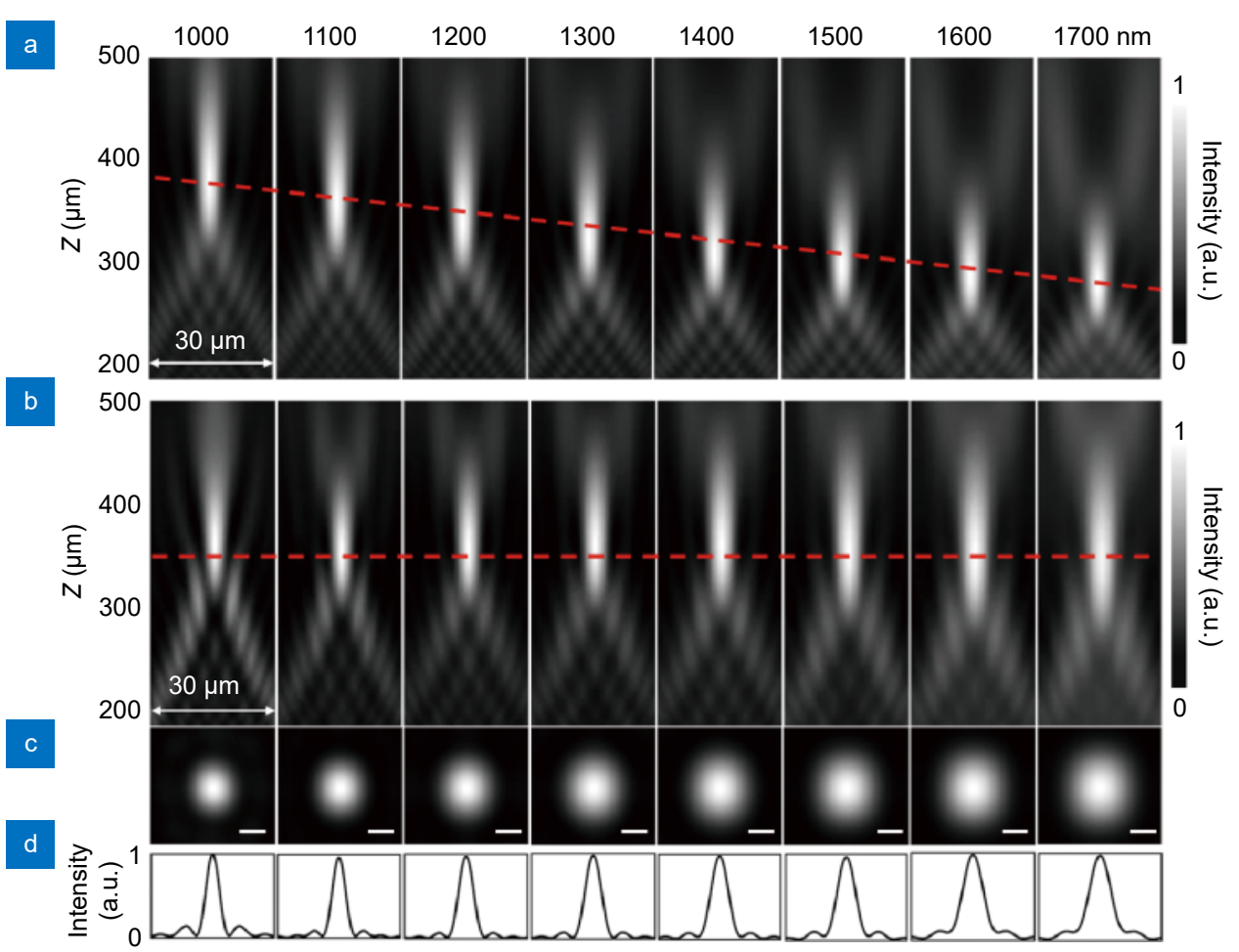

Fig. 4 | Simulated verification of chromatic and achromatic metalens. (a, b) Numerical intensity profiles of broadband chromatic (a) and achromatic (b) metalens with NA $=0.15$ at various incident wavelengths. The red dashed line indicates the position of the focal plane. (c, d) Normalized intensity profiles along the red dashed lines of (b). Scale bar: $6 \mu \mathrm{m}$.
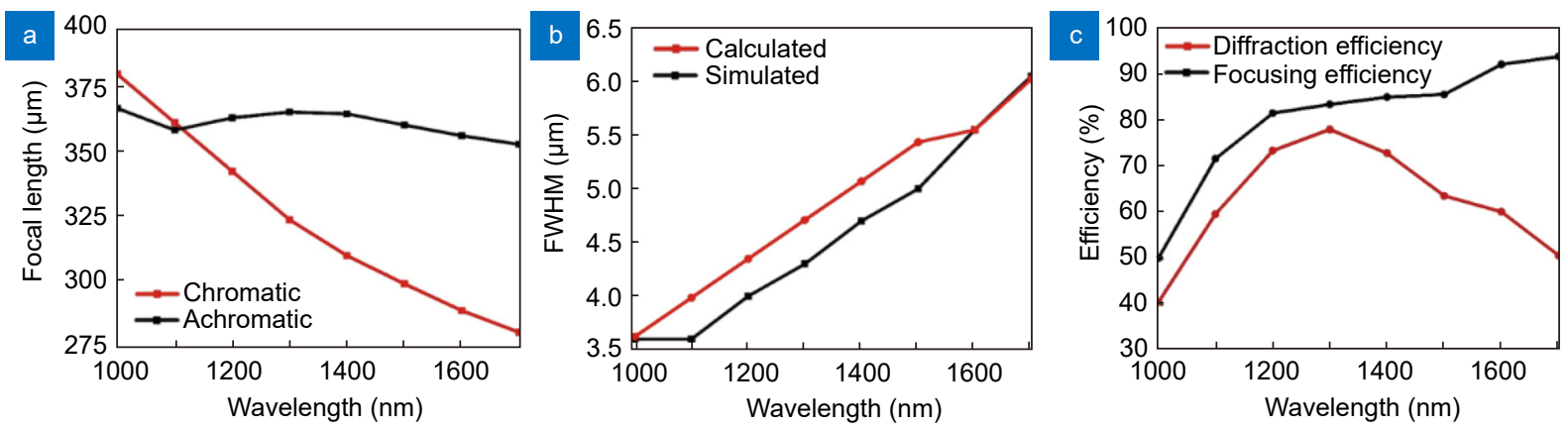

Fig. 5 | Performance of broadband achromatic metalens. (a) The focal length shift values of both chromatic and achromatic metalenses as a function of incident wavelength. (b) The FWHM of the focal spots as a function of indent wavelength. (c) The efficiency of achromatic metalens as a function of incident wavelength.

achromatic metalens, Fig. 5(a) shows the focal length shift values of both chromatic and achromatic metalenses as a function of incident wavelength. For the wavelength ranging from $1000 \mathrm{~nm}$ to $1700 \mathrm{~nm}$, the focal length shifts more than $100 \mu \mathrm{m}$ for the chromatic metalens while keeps almost invariable for the bilayer achromatic metalens. As a result, the bilayer metalens achieves the achromatic bandwidth of $700 \mathrm{~nm}$, which is about $52 \%$ relative to the central wavelength and larger than that of the single layer one. Fig. 5(b) shows the fullwidth half-maximum (FWHM) of the focal spots of the bilayer metalens. It can be seen that the simulated
FWHM values are close to the theoretical diffractionlimited values $\left(\frac{\lambda}{2 N A}\right)$ of the designed metalens with $\mathrm{NA}=0.15$. In addition to the achromatic bandwidth, the bilayer metalens also has high diffraction and focusing efficiency, as shown in Fig. 5(c). The focusing efficiency is defined as the ratio of light intensity from the focal spot to the light intensity of incident beam. Due to the employment of low loss $\mathrm{Si}$ as constituent material and the optimization of the geometry parameters for the nanopillar structures, the bilayer metalens has the averaged diffraction and focusing efficiencies about $81 \%$ and $64 \%$ for the investigated wavelength range, respectively, and 


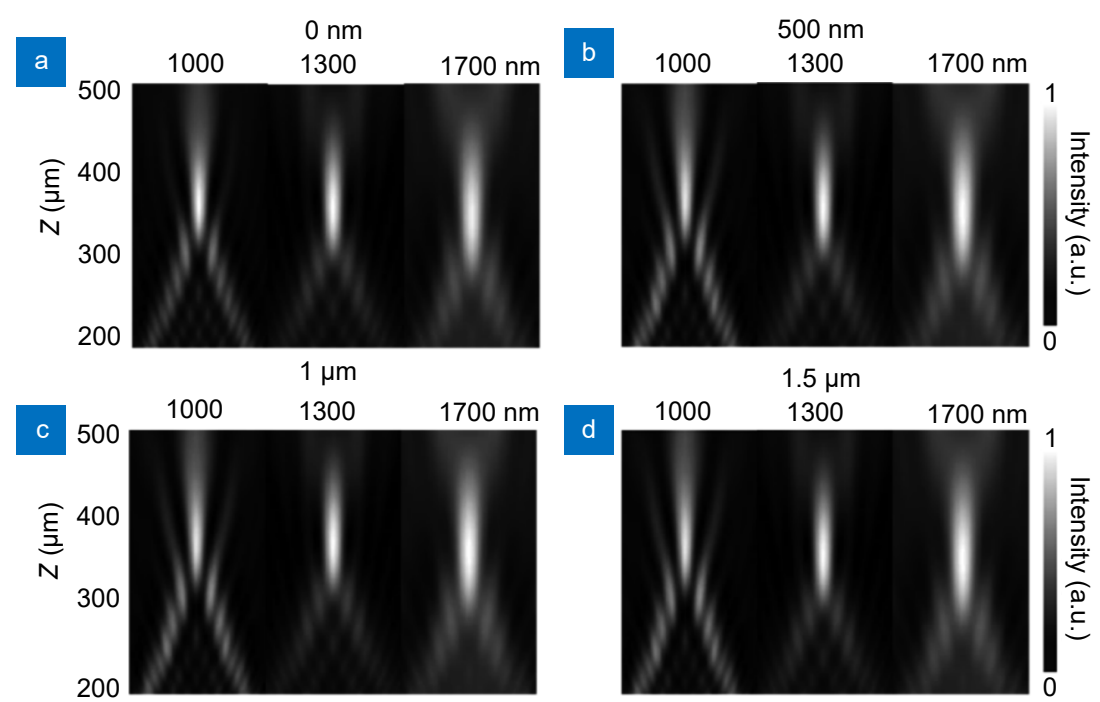

Fig. 6 | The influence of structural deviations on the lensing quality. (a) - (d) The simulated focal intensity profiles of bilayer metalens with perfect alignment (a), misalignment of $500 \mathrm{~nm}$ (b), $1 \mu \mathrm{m}$ (c) and $1.5 \mu \mathrm{m}$ (d) between two metasurface layers at three different wavelengths.

the focusing efficiency can research up to $78 \%$ around the wavelength of $1300 \mathrm{~nm}$. The focusing efficiency decreases quickly at the wavelength longer than $1400 \mathrm{~nm}$, which is mainly attributed to the lower polarization conversion efficiency of the nanopillar. Moreover, although similar efficiency of achromatic metalens has been reported in previous work ${ }^{40}$, it has much larger diameter while our design is more feasible for practical imaging applications. These results imply that the bilayer achromatic metalens proposed here have good performance of correcting chromatic aberration over a continuous wide range of near-infrared wavelength. Our method can also be used to design the high efficiency achromatic metalens with large diameter or high numerical aperture.

Finally, considering the alignment tolerance between two metasurface layers during the fabrication of bilayer metalens, we further investigate the influence of structural deviations on the lensing quality. Figs. 6(a)- 6(d) shows the simulated focal intensity profiles of bilayer metalens with perfect alignment, misalignment of 500 $\mathrm{nm}$ (one unit cell), $1 \mu \mathrm{m}$ (two unit cells) and $1.5 \mu \mathrm{m}$ (three unit cells) between two metasurface layers at three different wavelengths. It can be clearly seen that the all the cases have similar focal intensity profiles and the focal lengths are almost the same. This originates from the fact that the required focusing phase are from the top metasurface layer while the bottom metasuface layer mainly provides dispersion compensation. Therefore, this proposed bilayer achromatic metalens has a robust tolerance for nanofabrication.

\section{Conclusion}

In summary, we propose a new approach to design high efficiency achromatic metalens with large operation bandwidth by the modulation of dispersion using bilayer architecture. Two stacked metasufaces layers are designed to separately provide required focusing phase profile and dispersion compensation. As a proof-ofconcept demonstration, by using Si rectangular and cylindrical nanopillar arrays, we design a broadband achromatic metalens with $700 \mathrm{~nm}$ operation bandwidth and $64 \%$ averaged focusing efficiency working at nearinfrared region. Compared with conventional achromatic multilevel diffractive elements ${ }^{41}$, our device provides a general approach for achieving multifunctional high pixel density achromatic optics. This method solves the problem of mutual constraint between required phase dispersion and efficiency in single layer metasurface and opens up the possibility to design multifunctional broadband meta-devices.

\section{References}

1. Pedrotti FL, Pedrotti LS. Introduction to Optics (Prentice-Hall, Englewood Cliffs, N.J., 1987).

2. Yu NF, Genevet P, Kats MA, Aieta F, Tetienne JP et al. Light propagation with phase discontinuities: Generalized laws of reflection and refraction. Science 334, 333-337 (2011).

3. Kildishev AV, Boltasseva A, Shalaev VM. Planar photonics with metasurfaces. Science 339, 1232009 (2013).

4. Lin DM, Fan PY, Hasman E, Brongersma ML. Dielectric gradient metasurface optical elements. Science $\mathbf{3 4 5}, \mathbf{2 9 8 - 3 0 2}$ (2014).

5. Arbabi A, Horie Y, Bagheri M, Faraon A. Dielectric metasurfaces for complete control of phase and polarization with subwavelength spatial resolution and high transmission. Nat 
Nanotechnol 10, 937-943 (2015).

6. Ding XM, Monticone F, Zhang K, Zhang L, Gao DL et al. UItrathin Pancharatnam-Berry metasurface with maximal crosspolarization efficiency. Adv Mater 27, 1195-1200 (2015).

7. Li ZY, Kim MH, Wang C, Han ZH, Shrestha S et al. Controlling propagation and coupling of waveguide modes using phasegradient metasurfaces. Nat Nanotechnol 12, 675-683 (2017).

8. Yue FY, Zhang CM, Zang XF, Wen DD, Gerardot BD et al. High-resolution grayscale image hidden in a laser beam. Light Sci App/ 7, 17129 (2018).

9. Divitt S, Zhu WQ, Zhang C, Lezec HJ, Agrawal A. Ultrafast optical pulse shaping using dielectric metasurfaces. Science $\mathbf{3 6 4}$, 890-894 (2019).

10. Ma XL, Pu MB, Li X, Guo YH, Luo XG. All-metallic wide-angle metasurfaces for multifunctional polarization manipulation. Opto-Electron Adv 2, 180023 (2019).

11. Guo JY, Wang T, Quan BG, Zhao H, Gu CZ et al. Polarization multiplexing for double images display. Opto-Electron Adv $\mathbf{2}$, 180029 (2019).

12. Dou KH, Xie X, Pu MB, Li X, Ma XL et al. Off-axis multiwavelength dispersion controlling metalens for multi-color imaging. Opto-Electron Adv 3, 190005 (2020).

13. Ni XJ, Kildishev AV, Shalaev VM. Metasurface holograms for visible light. Nat Commun 4, 2807 (2013).

14. Zheng GX, Mühlenbernd H, Kenney M, Li GX, Zentgraf T et al. Metasurface holograms reaching $80 \%$ efficiency. Nat Nanotechnol 10, 308-312 (2015).

15. Huang K, Dong ZG, Mei ST, Zhang L, Liu YJ et al. Silicon multi-meta-holograms for the broadband visible light. Laser Photonics Rev 10, 500-509 (2016).

16. Li X, Chen LW, Li Y, Zhang XH, Pu MB et al. Multicolor 3D meta-holography by broadband plasmonic modulation. Sci $\mathrm{AdV}$ 2, e1601102 (2016).

17. Li LL, Cui TJ, Ji W, Liu S, Ding J et al. Electromagnetic reprogrammable coding-metasurface holograms. Nat Commun 8 , 197 (2017).

18. Arbabi E, Kamali SM, Arbabi A, Faraon A. Vectorial holograms with a dielectric metasurface: ultimate polarization pattern generation. ACS Photonics 6, 2712-2718 (2019).

19. Feng $\mathrm{H}, \mathrm{Li}$ QT, Wan WP, Song JH, Gong QH et al. Spin-switched three-dimensional full-color scenes based on a dielectric meta-hologram. ACS Photonics 6, 2910-2916 (2019).

20. Khorasaninejad M, Chen WT, Devlin RC, Oh J, Zhu AY et al. Metalenses at visible wavelengths: Diffraction-limited focusing and subwavelength resolution imaging. Science 352, 1190-1194 (2016).

21. Arbabi E, Arbabi A, Kamali SM, Horie Y, Faraji-Dana MS et al. Mems-tunable dielectric metasurface lens. Nat Commun $\mathbf{9}$, 812 (2018).

22. Yang ZY, Wang ZK, Wang YX, Feng $X$, Zhao $M$ et al. Generalized hartmann-shack array of dielectric metalens sub-arrays for polarimetric beam profiling. Nat Commun 9, 4607 (2018).

23. Pors A, Nielsen MG, Bozhevolnyi SI. Plasmonic metagratings for simultaneous determination of stokes parameters. Optica $\mathbf{2}$, 716-723 (2015).

24. Chen WT, Török P, Foreman MR, Liao CY, Tsai WY et al. Integrated plasmonic metasurfaces for spectropolarimetry. Nanotechnology 27, 224002 (2016).

25. Aieta F, Kats MA, Genevet P, Capasso F. Multiwavelength achromatic metasurfaces by dispersive phase compensation. Science 347, 1342-1345 (2015).

26. Wang B, Dong FL, Li QT, Yang D, Sun CW et al. Visible-frequency dielectric metasurfaces for multiwavelength achromatic and highly dispersive holograms. Nano Lett 16, 5235-5240 (2016).

27. Arbabi E, Arbabi A, Kamali SM, Horie Y, Faraon A. Multiwavelength polarization-insensitive lenses based on dielectric metasurfaces with meta-molecules. Optica 3, 628-633 (2016).

28. Khorasaninejad M, Shi Z, Zhu AY, Chen WT, Sanjeev V et al. Achromatic metalens over $60 \mathrm{~nm}$ bandwidth in the visible and metalens with reverse chromatic dispersion. Nano Lett 17, 1819-1824 (2017).

29. Arbabi E, Arbabi A, Kamali SM, Horie Y, Faraon A. Controlling the sign of chromatic dispersion in diffractive optics with dielectric metasurfaces. Optica 4, 625-632 (2017).

30. Avayu O, Almeida E, Prior P, Ellenbogen T. Composite functional metasurfaces for multispectral achromatic optics. Nat Commun 8, 14992 (2017).

31. Zhou Y, Kravchenko II, Wang H, Nolen JR, Gu G et al. Multilayer noninteracting dielectric metasurfaces for multiwavelength metaoptics. Nano Lett 18, 7529-7537 (2018).

32. Wang SM, Wu PC, Su V, Lai YC, Chu CH et al. Broadband achromatic optical metasurface devices. Nat Commun 8, 187 (2017).

33. Chen WT, Zhu AY, Sanjeev V, Khorasaninejad M, Shi ZJ et al. A broadband achromatic metalens for focusing and imaging in the visible. Nat Nanotechnol 13, 220-226 (2018).

34. Wang SM, Wu PC, Su VC, Lai YC, Chen MK et al. A broadband achromatic metalens in the visible. Nat Nanotechnol 13, 227-232 (2018).

35. Shrestha S, Overvig AC, Lu M, Stein A, Yu NF. Broadband achromatic dielectric metalenses. Light Sci App/ 7, 85 (2018).

36. Chen WT, Zhu AY, Sisler J, Huang YW, Yousef KMA et al. Broadband achromatic metasurface-refractive optics. Nano Lett 18, 7801-7808 (2018).

37. Hsiao HH, Chen YH, Lin RJ, Wu PC, Wang SM et al. Integrated resonant unit of metasurfaces for broadband efficiency and phase manipulation. Adv Optical Mater 6, 1800031 (2018).

38. Fan ZB, Qiu HY, Zhang HL, Pang XN, Zhou LD et al. A broadband achromatic metalens array for integral imaging in the visible. Light Sci App/ 8, 67 (2019).

39. Chen WT, Zhu AY, Sisler J, Bharwani Z, Capasso F. A broadband achromatic polarization-insensitive metalens consisting of anisotropic nanostructures. Nat Commun 10, 355 (2019).

40. Chung H, Miller OD. High-NA achromatic metalenses by inverse design. arXiv preprint arXiv: 1905.09213 (2019).

41. Banerji S, Meem M, Majumder A, Dvonch C, Sensale-Rodriguez $B$ et al. Single flat lens enabling imaging in the short-wave infra-red (SWIR) band. OSA Contin 2, 2968-2974 (2019).

\section{Acknowledgements}

We are grateful for financial supports from National Natural Science Foundation of China (11774163) and Fundamental Research Funds for Central Universities.

\section{Competing interests}

The authors declare no competing financial interests. 